Accepted Manuscript

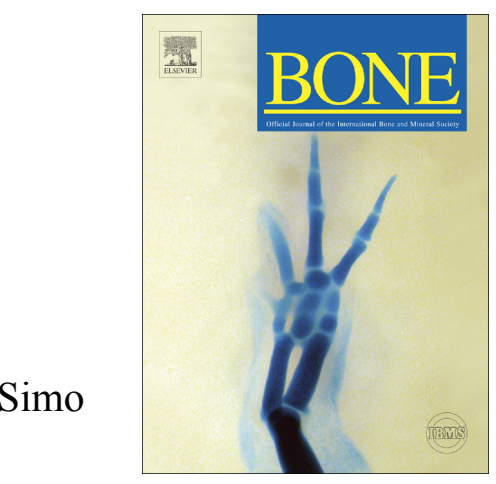

Structural risk factors for low-energy acetabular fractures

Robel K. Gebre, Jukka Hirvasniemi, Iikka Lantto, Simo Saarakkala, Juhana Leppilahti, Timo Jämsä

PII:

S8756-3282(19)30278-9

DOI: https://doi.org/10.1016/j.bone.2019.07.004

Reference:

BON 12028

To appear in:

Bone

Received date:

5 March 2019

Revised date:

28 June 2019

Accepted date:

4 July 2019

Please cite this article as: R.K. Gebre, J. Hirvasniemi, I. Lantto, et al., Structural risk factors for low-energy acetabular fractures, Bone, https://doi.org/10.1016/ j.bone.2019.07.004

This is a PDF file of an unedited manuscript that has been accepted for publication. As a service to our customers we are providing this early version of the manuscript. The manuscript will undergo copyediting, typesetting, and review of the resulting proof before it is published in its final form. Please note that during the production process errors may be discovered which could affect the content, and all legal disclaimers that apply to the journal pertain. 


\section{STRUCTURAL RISK FACTORS FOR LOW-ENERGY ACETABULAR FRACTURES}

Robel K. Gebre ${ }^{1}$, Jukka Hirvasniemi ${ }^{2}$, Iikka Lantto ${ }^{3,4}$, Simo Saarakkala ${ }^{1,4,5}$, Juhana Leppilahti ${ }^{3,4}$, Timo Jämsä ${ }^{1,4,5}$

${ }^{1}$ Research Unit of Medical Imaging, Physics and Technology, University of Oulu, Oulu, Finland

${ }^{2}$ Department of Radiology \& Nuclear Medicine, Erasmus University Medical Center, Rotterdam, the Netherlands

${ }^{3}$ Division of Orthopaedic and Trauma Surgery, Oulu University Hospital, Oulu, Finland

${ }^{4}$ Medical Research Center, University of Oulu and Oulu University Hospital, Oulu, Finland

${ }^{5}$ Diagnostic Radiology, Oulu University Hospital, Oulu, Finland

Corresponding author: Robel K. Gebre

Contact address: Research Unit of Medical Imaging, Physics and Technology, University of Oulu, Oulu, Finland. Phone number: +358414747822, Email: robel.gebre@ oulu.fi.

Declarations of interest: none 


\section{ABSTRACT}

In this study, we aimed to clarify proximal femur and acetabular structural risk factors associated with low-energy acetabular fractures in the elderly using three-dimensional (3D) computed tomography (CT). Pelvic bones and femurs were segmented and modeled in 3D from abdominopelvic CT images of 121 acetabular fracture patients (mean age $72 \pm 12$ years, range $50-98$ years, 31 females and 90 males) and 121 age-gender matched controls with no fracture. A set of geometric parameters of the proximal femur and the acetabulum was measured. An independentsamples t-test or a Mann-Whitney U-test was used for statistical analyses. The fractured side was used for proximal femur geometry, while the contralateral side was used for acetabular geometry. The neck shaft angle (NSA) was significantly smaller (mean $122.1^{\circ}\left[95 \%\right.$ CI $121.1^{\circ}-123.2^{\circ}$ ] vs. $\left.124.6^{\circ}\left[123.6^{\circ}-125.6^{\circ}\right], p=0.001\right)$ and the femoral neck axis length (FNALb) was significantly longer $(78.1 \mathrm{~mm}[77.0-79.2 \mathrm{~mm}]$ vs. $76.0 \mathrm{~mm}[74.8-77.2 \mathrm{~mm}], p=0.026)$ in the fracture group than in the controls when genders were combined. The NSA was significantly smaller both for females $\left(120.2^{\circ}\left[117.8^{\circ}-122.6^{\circ}\right]\right.$ vs. $\left.124.7^{\circ}\left[122.5^{\circ}-127.0^{\circ}\right], p=0.007\right)$ and for males $\left(122.7^{\circ}\right.$ $\left[121.5^{\circ}-123.8^{\circ}\right]$ vs. $\left.124.6^{\circ}\left[123.4^{\circ}-125.7^{\circ}\right], p=0.006\right)$ in the fracture group. However, only males showed a significantly longer FNALb $(80.0 \mathrm{~mm}[78.9-81.1 \mathrm{~mm}]$ vs. $77.8 \mathrm{~mm}[76.6-79.0 \mathrm{~mm}], p=$ 0.025). No statistically significant associations of acetabular geometry with fractures were found. However, the mean values of the acetabular angle of Sharp $\left(34^{\circ}\right)$, the lateral center-edge angle $\left(40^{\circ}\right)$, the anterior center-edge angle $\left(62^{\circ}\right)$, and the posterior center-edge angle $\left(105^{\circ}\right)$ indicated possible over-coverage. In conclusion, our findings suggest that proximal femur geometry is associated with low-energy acetabular fractures. Especially elderly subjects with an NSA smaller than normal have an increased risk of acetabular fractures.

KEY WORDS: acetabular fracture; three-dimensional computed tomography; structural risk factors; proximal femur geometry; acetabular geometry 


\section{Introduction}

Falls from standing height are the primary injury mechanisms for low-energy acetabular fractures in the elderly [1-3]. A 2.4-fold increase in the incidence of low-energy pelvic and acetabular fractures in the elderly over the last three decades has been reported both in Finland and in the United States of America [4,5]. The most common fracture patterns resulting from sideways falls are anterior column and anterior column-posterior hemi-transverse fracture patterns [1,2]. The contributing factors for specific fracture patterns are the mechanics of injury, bone density, and the orientation of the pelvis and femur during a fall $[2,5,6]$. The mechanics of injury refers to the sideways impacts, which transmit forces through the greater trochanter to realign the femur anteromedially with the anterior column of the acetabulum [1-3].

Pelvic geometry can be measured in two-dimensions (2D) from, e.g., anterior-posterior (a.p) plain radiography or computed tomography (CT) slices or in three-dimensions (3D) from CT reconstruction models. Measurement of pelvic geometry using traditional 2D methods is somewhat challenging due to the complex anatomy of the pelvis. In 2D studies, the orientation of subjects during scanning needs to be carefully controlled in order to minimize the effects of tilting and rotation of the femur and pelvis. Especially images collected from clinical databases in which patient positioning may not have been appropriately standardized can have oblique pelvic tilting and femur head rotation. Instead, 3D methods can overcome these challenges by generating models from CT slices that can be freely rotated and reoriented.

Usually, proximal femur geometry (PFG) is defined by the neck shaft angle (NSA), femoral neck axis length (FNAL), hip axis length (HAL), femoral head diameter (FHD), and neck shaft width (NSW). Standardized normal values of PFG variables have clinical significance in, e.g., preoperative planning and identifying or predicting risk factors for hip fractures [7-9]. However, 2D 
methods give only projected values, and femur rotation, e.g., cannot easily be determined from these values. Consequently, Boese et al. found significant differences in NSAs taken from a.p CT slices compared with coronal reconstruction slices rotated on the femoral neck planes [10]. Further, Anastopoulos et al. also compared 3D true and projected values of NSAs from 3D CT models and found a difference between the two methods [7]. It is worth noting that the average rotated NSA reported by Boese et al. was greater than the true 3D mean values reported by Anastopoulos et al $[7,10]$.

Acetabular geometry (AG) describes the orientation of the acetabulum on the pelvis and its relationship to the femur. Acetabular coverage and support of the femur head provide biomechanical and diagnostic information about the hip joint. In clinical practice, a.p plain radiography and $\mathrm{CT}$ are commonly used to diagnose different joint conditions. However, AG 2D methods are prone to several challenges such as identifying clear landmarks and pelvic tilting and rotation [11-14]. Discrepancies in acetabular coverage variables with bony-edge vs. sourcil-edge measurements as landmarks are instances in which landmark variations occur [11-13]. Moreover, central beam offset variations, pelvic tilting and rotation may impede the accuracy of 2D methods $[15,16]$. On the contrary, with $3 \mathrm{D}$ methods, the pelvis can be freely rotated to a redefined alignment position, and a new 3D coordinate system can be created using the anatomical landmarks. With 3D methods, annotation of anatomical landmarks is less challenging than with 2D methods. Most commonly, bilateral anterior superior iliac spines (ASISs) and pubic tubercles (PTs) are used to define an anterior pelvic plane (APP), from which the remaining two orthogonal planes, the midsagittal plane (MSP) and the trans-pelvic plane (TPP), are drawn [17-19]. Once the pelvic 3D coordinate system is created, it can be reoriented and aligned with a global coordinate system to standardize all measurements. 
Previously, PFG variables, mainly the NSA and the FNAL, have been shown to be significantly different for proximal femur fractures when compared with controls $[9,20]$. Similarly, AG variables are commonly used to identify hip abnormalities such as hip dysplasia and over-coverage, which can lead to hip osteoarthritis (OA) [21-24]. However, there are no 3D studies that give insight into the association of these variables with low-energy acetabular fractures. Therefore, our aim was to identify PFG and AG variables that contribute to the risk of acetabular fractures, using clinical 3D CT images of elderly subjects who had sustained acetabular fractures and those of their age-gender matched controls.

\section{Materials and Methods}

\subsection{Study Subjects}

The data consisted of abdominopelvic CT images of subjects with acetabular fractures $(n=121)$ and their age-gender matched controls $(n=121)$. The images were obtained from the picture archiving and communication system (PACS) of Oulu University Hospital, Oulu, Finland. The images were taken from patients admitted between January 2008 and October 2017. A research permit (number 220/2017) was obtained from the Northern Ostrobothnia Hospital District. Written informed consent was not required due to the register-based study design. The exclusion criteria were age (minimum age 50 years), type of trauma, femur fractures, or previous pelvic diseases.

The data comprised a total of 242 subjects (age range 50-98 years) of which $26 \%$ were females $(n=$ $62)$ and $74 \%$ were males $(n=180)$ (Figure 1). PFG and AG were defined for the same subjects. For comparison of mean differences, PFG was measured on the fracture side and AG on the contralateral side in the fracture cases, and on the matching sides for the controls. In addition, the contralateral side in the fracture cases was used for PFG in the correlation analyses. Subjects containing two-sided acetabular fractures were excluded from the AG analysis $(n=22)$. However, 
they were included in the PFG analysis. Images with insufficient quality (e.g., artifacts, structures only partly visible in the image) were also excluded from the PFG $(n=12)$ and AG analyses $(n=$ 4). Thus, the final dataset contained 230 subjects $(n=55$ females: mean age (standard deviation, or SD): 77.0 (13.0) years; $n=175$ males: 70.5 (11.9) years) for the PFG analysis, and 216 subjects $(n$ $=57$ females: 77.6 (13.4) years; $n=159$ males; 71.2 (10.8) years) for the AG analysis.

\section{2. $3 D$ modeling}

Thresholding, segmentation, and 3D modeling of the CT data were done using Mimics (Materialise, Leuven BE, Belgium) software. CT slice thicknesses varied from 0.5 to $10.0 \mathrm{~mm}$ and pixel sizes (isotropic) from 0.3 to $1.1 \mathrm{~mm}$. Bone and soft tissue boundaries were first segmented using thresholding mask windows based on Hounsfield units (HU). A predefined window in the Mimics, starting at a lower boundary of $200 \mathrm{HU}$, was used to exclude soft tissues and to segment bone tissue. These masks were applied to each slice and then separated into pelvic and femur masks. Each slice was visually examined for segmentation and any overlap of masks. Discontinuous edges and gaps in the masks were corrected with dilation morphological operations to create uniform masks with well-defined edges. Finally, each mask was optimally reconstructed into a 3D model, which was exported into 3-Matic (Materialise, Leuven BE, Belgium) for geometry measurement. Postprocessing, such as meshing, smoothing, and wrapping, was not performed on the models so as not to affect the dimensions.

\subsection{Proximal femur geometry $(P F G)$ analysis}

PFG variables consisted of the NSA, FNALa, FNALb, NSW, and FHD, as shown in Figure 2. The NSA was defined as the angle between the medial axes of the femur shaft and neck. To measure the true 3D NSA, the femur shaft axis was created through the middle of the proximal canal [7]. The neck shaft axis was created through the neck's narrowest midsection passing through the femur 
head center. The femoral neck length was measured using two different methods, i.e., FNALa and FNALb $[9,25,26]$. In both methods, the starting point was the intersection point of the neck axis below the greater trochanter. The FNALa was measured up to the anterior point where the neck axis exits the femur head, and the FNALb was measured up to the center [9]. The NSW was the diameter of the femoral neck at its narrowest midsection. The fitting function in 3-Matic was used to calculate the axes lines and to approximate a sphere around the femur head and the circle that fits the midsection of the neck.

\subsection{Acetabular geometry (AG) analysis}

Before acetabular geometry was measured, the pelvis was realigned in order to correct the pelvic rotation introduced at the time of scanning and to standardize measurements across the data set, as shown in Figure 3b. The resulting coordinate system consisted of three orthogonal planes: the anterior pelvic plane (APP), mid-sagittal plane (MSP), and trans-pelvic plane (TPP), as shown in Figure 3a. Four anatomical landmarks, i.e., right and left bilateral anterior superior iliac spines (ASIS) and pubic tubercles (PT), were used to create the APP [17,18]. Landmarks were located using the extrema analysis function in 3-Matic, which calculates extrema points along an axis. In this case, the $y$-axis of the global coordinate system was used as the analysis direction. The APP together with the 3D model was then aligned to the frontal plane (XZ-plane) of the global coordinate system. The remaining two planes, the MSP and the TPP, were placed perpendicular to the APP to form the new coordinate system. Following realignment and reorientation of the 3D model, acetabular orientation, coverage, and support were measured as follows.

\subsubsection{Acetabular orientation}

Acetabular orientation was defined by acetabular inclination (AI) and anteversion (AceAV). First, a plane and a perpendicular line to that plane were fitted to the acetabular rim using marking and 
plane fitting functions in 3-Matic. The plane is the acetabular rim plane, and the perpendicular line is known as the acetabular axis [27]. According to the definitions set by Murray [27], radiographic orientation was used to measure AceAV and AI on the APP and on the longitudinal axis of the MSP, respectively, as shown in Figure 3a [17,18,27].

\subsubsection{Acetabular coverage}

Acetabular coverage was defined by four variables: the acetabular angle of Sharp (AA), lateral center-edge angle (LCEA), anterior center-edge angle (ACEA), and posterior center-edge angle (PCEA) (Figure 4). The AA and the LCEA were measured on the APP (Figure 4a). The AA has its vertex on the bony ridges of the acetabular fossa and is subtended by a horizontal line and a line tangential to the lateral edge of the acetabular roof [28]. The LCEA is the angle between a vertical line and a line from the center of the femoral head to the lateral margin of the acetabulum [11]. The ACEA and the PCEA are measured on the MSP. Two lines are formed between the center of the femoral head to the anterior and posterior acetabular margins, respectively. The ACEA is the angle between a vertical line through the center of a femoral head and the anterior margin line. The PCEA is the angle between a vertical line through the center of a femoral head and the posterior margin line (Figure 4c) [18].

\subsubsection{Acetabular Support}

Acetabular support information was provided by the anterior sector angle (ASA) and the posterior sector angle (PSA), and they were measured on the TPP. The ASA is the angle between a vertical line and a line through the center of a femoral head to the anterior acetabular margin. The PSA is the angle between a vertical line and a line through the center of a femoral head to the posterior acetabular margin (Figure 4b). An equatorial plane that passes through both femoral head centers was not possible because of the misalignment of the heads caused by the fractures. Anda et al. have 
used a similar procedure for subjects that had oblique right-left femur positions [14]. Consequently, since patient positioning was not controlled, we applied a standard procedure of measuring through just one femoral head center in the entire data set.

\subsection{Statistical Analysis}

A Shapiro-Wilk test was performed to determine the distribution of each variable prior to statistical analyses. For normality, the null hypothesis was rejected at $p<0.05$. Following the normality test, a statistical analysis was conducted using an independent-samples t-test or a Mann-Whitney U-test for normally and not normally distributed data, respectively. Statistical analysis for proximal femur geometry was done on the fractured side, while for acetabular geometry the analysis was conducted on the contralateral side of the fracture. A bivariate Pearson's linear correlation analysis was performed to investigate the association between PFG and AG as well as between variables within each geometry. Because AG was measured on the contralateral side of a fracture, the correlation and mean differences between the right and left sides of PFG and AG variables were checked on a randomly selected subset of control subjects ( $n=19,5$ females and 14 males) to assess the relation between right and left sides. The IBM SPSS (version 24.0.0.1, Armonk, CINY: IBM Corp, USA) statistics program was used for the statistical analyses.

\subsection{Interrater reliability and intrarater repeatability}

In order to check the repeatability and reliability of the methods used, a two-way mixed intraclass correlation coefficient (ICC) was calculated for randomly selected cases $(n=20,10$ fractures and 10 controls). The intrarater repeatability was checked using values analyzed three times by one rater $(\mathrm{RG})$, with measurements taken one week apart; the interrater reliability was a single-measure test between two raters $(\mathrm{RG}, \mathrm{JH})$. 


\section{Results}

\subsection{Intrarater reliability and intrarater repeatability}

Strong intrarater repeatability and interrater reliability of the PFG and AG measurements were found. The ICCs (intrarater, interrater) for the different PFG and AG variables ranged from (0.84 to $0.96,0.89$ to 0.97$)$ and $(0.96$ to $0.98,0.97$ to 0.99$)$, respectively (Table 1$)$.

\subsection{Fractures vs Controls}

Among the fracture and control groups without gender classification, the NSA was significantly smaller in the fracture group than in the control group (mean $[95 \% \mathrm{CI}]: 122.1^{\circ}\left[121.1^{\circ}-123.2^{\circ}\right]$ vs. $\left.124.6^{\circ}\left[123.6^{\circ}-125.6^{\circ}\right], p=0.001\right)$. The FNALb was significantly longer in the fracture group than in the control group $(78.1 \mathrm{~mm}[77.0 \mathrm{~mm}-79.2 \mathrm{~mm}]$ vs. $76.0 \mathrm{~mm}[74.8 \mathrm{~mm}-77.2 \mathrm{~mm}], p=$ 0.026) (Table 2).

The NSA was significantly smaller in the fracture group than in the control group, both in females $\left(120.2^{\circ}\left[117.8^{\circ}-122.6^{\circ}\right]\right.$ vs. $\left.124.7^{\circ}\left[122.5^{\circ}-127.0^{\circ}\right], p=0.007\right)$ and in males $\left(122.7^{\circ}\left[121.5^{\circ}-\right.\right.$ $\left.123.8^{\circ}\right]$ vs. $\left.124.6^{\circ}\left[123.4^{\circ}-125.7^{\circ}\right], p=0.006\right)$. However, only males showed significantly longer FNALbs in the fracture group than in the control group $(80.0 \mathrm{~mm}[78.9 \mathrm{~mm}-81.1 \mathrm{~mm}] \mathrm{vs} .77 .8$ $\mathrm{mm}[76.6 \mathrm{~mm}-79.0 \mathrm{~mm}], p=0.025)$.

\subsection{Associations between geometrical variables}

In the assessment of the relationships between the different PFG variables, the FNALa and FNALb were found to be very strongly correlated $(R=0.96, p<0.01)$. Except for the NSA, the remaining PFG variables were positively and statistically significantly correlated with each other (Table 3).

In the assessment of the relationships between the different AG variables, AceAV was found to be negatively correlated with the ASA $(R=-0.58, p<0.01)$ and the ACEA $(R=-0.62, p<0.01)$ and 
positively correlated with the PSA $(R=0.63, p<0.01)$ and the PCEA $(R=0.62, p<0.01)$. The LCEA was positively correlated with the ASA $(R=0.51, p<0.01)$ and the ACEA $(R=0.49, p<$ $0.01)$. The ACEA was strongly correlated with the ASA $(R=0.71, p<0.01)$, and the PCEA was strongly correlated with the PSA $(R=0.83, p<0.01)$ (Table 3$)$.

Only weak correlations were found between the PFG and AG variables (Table 3).

\subsection{Right vs Left}

In the assessment of the associations between the PFG and AG variables of the right and left sides, all variables were found to show a strong correlation $(R>0.8, p<0.01)$ except for the NSA $(R=$ $0.53, p<0.01)$. There were no statistically significant differences in PFG and AG variables between the right and left sides except for the NSA (Table 4). 


\section{Discussion}

Acetabular and femur geometry have previously been studied using plain radiography and 2D CT methods in relation to hip fractures, hip dysplasia, and osteoarthritis [21-23,29,30]. However, there are no 3D CT studies on the etiology of low-energy acetabular fractures in the elderly. In the present study, we for the first time measured acetabular and proximal femur geometry in 3D to find variables associated with acetabular fractures. Our findings suggest that a smaller neck shaft angle is indicative of acetabular fractures in both genders. Previously, it has been reported that subjects with femoral neck fractures have larger NSAs compared with trochanteric fractures or controls $[9,20]$. This suggests that a Varus hip $\left(\mathrm{NSA} \leq 120^{\circ}\right)$ may transfer the impact load via the femoral head to the acetabulum, which may lead to an acetabular fracture. The mean NSA for nonfracture and contralateral sides was $125^{\circ}$, which is smaller than the 2D CT NSA $\left(129^{\circ}\right)$ measured by Boese et al. but closer to the 3D NSA $\left(123^{\circ}\right)$ reported by Anastopoulos et al $[7,10]$.

We also found that a longer femoral neck axis length (FNALb) is indicative of acetabular fractures in males but not in females. Furthermore, there was a trend that the other femoral neck axis variable, FNALa, was longer in male fracture cases than in controls. These findings might be related to impact mechanics. Both the FNALa and the FNALb represent the length of the femoral neck axis, with the FNALb measured up to the femoral head center and the FNALa defined to reach the anterior site where the neck axis exits on the surface of the femoral head close to the acetabular wall. Consequently, the proximity of the FNALa to the impact site with the acetabular wall might have caused a slight shortening/or compressing of the femoral neck.

In this study, none of the acetabular geometry variables was found to be associated with fractures in either gender. With regards to acetabular orientation, mean acetabular inclination (AI) and anteversion (AceAV) were $51^{\circ}$ and $14^{\circ}$ respectively. These results closely match previous findings 
reported by Higgins et al. [17], Wang et al. [31], and Zhang et al. [19] as normal values for AI and AceAV $\left(54^{\circ}, 19^{\circ}\right),\left(49^{\circ}, 14^{\circ}\right)$, and $\left(52^{\circ}, 16^{\circ}\right)$ respectively. In addition, AceAV was positively correlated with posterior coverage (PCEA) and support (PSA) variables but negatively correlated with anterior coverage (ACEA) and support (ASA) variables. This correlation pattern matches very closely the data by Anda et al., who made 2D CT measurements of acetabular anteversion and sector angles [14].

Direct measurement of $\mathrm{AG}$ on the fractured side was not possible due to dislocation gaps between fragments of the fractured acetabula. Hence, we measured AG on the contralateral side in the fracture cases, assuming symmetric acetabular geometry. To assess this, we checked correlation and mean differences in PFG and AG variables between right and left sides in a subset of control subjects, as shown in Table 4. Both AG and PFG variables had a strong side correlation, except for a side difference found in the NSA. Furthermore, a correlation analysis was performed between the PFG and AG variables taken from the contralateral side, as shown in Table 3. We found that the NSA and the FNALb were not strongly correlated with either the PFG or the AG variables. This suggests that the FNALb in males and the NSA in both genders are independent risk factors for an acetabular fracture.

Acetabular coverage of the femoral head was defined by the AA, LCEA, ACEA, and PCEA. These variables have been extensively studied using plain radiography, and their normal values are well defined for diagnosing acetabular over-coverage and hip dysplasia [22,32-34]. However, 2D CTbased methods have not been standardized, and the literature is limited for 3D studies $[34,35]$. We found the AA, LCEA, ACEA, and PCEA to be $35^{\circ}, 40^{\circ}, 62^{\circ}$, and $105^{\circ}$, respectively, which according to known normal radiographic values indicate over-coverage [22,32-34]. Furthermore, 
these values are consistent with the sex- and age-specific study by Miyasaka et al., in which subjects aged $\geq 50$ years showed over-coverage [33].

Acetabular support of the femur head is mainly provided by the sector angles ASA and PSA, which are used to diagnose hip dysplasia [14,23,33]. Our values matched previously reported values, except for the PSA in males, where there was a slight discrepancy [14,23]. The PSA was slightly lower $\left(96^{\circ}\right)$ than previously reported by Anda et al. $\left(102^{\circ}\right)$ and Tallroth et al. $\left(103^{\circ}\right)$, who did their measurements on 2D CT axial slices [14,23]. However, a similar discrepancy was observed by Suzuki et al. $\left(98^{\circ}\right)$, who conducted a 3D geometry study of acetabular orientation [18]. A possible explanation for variations in mean PSA values might be the differences in methodologies used. Nevertheless, both the ASA and the PSA values were in the normal range, as defined for 2D CT methods, and did not indicate hip dysplasia [14,33].

The PFG variables taken from the fractured sides gave us an insight into the role of femur geometry in acetabular fracture risk. From a biomechanical point of view, the proximal femur anatomical orientation might affect the joint stress/strain distribution, resulting in fracture patterns within the acetabulum. We found smaller NSAs in both genders and longer FNALbs in males as the main structural risk factors for acetabular fractures. Although we found no statistically significant association of acetabular morphology with fracture cases, we did observe some over-coverage. Here, AG was measured on the contralateral side, and determination of side-specific AG properties on the fractured side was not possible. Thus, whether over-coverage, hip dysplasia, hip OA, or other abnormalities contribute to acetabular fractures remains still unclear.

There were three major limitations of this study. First, different injury mechanisms and the relationship between acetabular fracture sub-types and geometry variables were not considered. The main injury mechanisms were low-energy fractures; however, there might have been some high- 
energy fractures mixed in the same group. Acetabular fractures are commonly classified into five elementary and five associated fracture types based on the method coined by Judet and Letournel [36]. Here, due to the limited sample of fracture subtype, all fracture cases were classified as one group. Previously, it has been shown that fracture patterns in the elderly are unique, but we did not test that observation in our study. Second, AG measurements were taken from the contralateral side, and obtaining side-specific information about acetabular geometry on the fracture side was not possible. The high correlation between right and left sides suggested that the contralateral side can be used for AG measurements. Lastly, a lack of standardized AG 3D values prevented us from making definite conclusions about the biomechanical role of acetabular orientation, coverage, and support in the incidence of acetabular fractures.

In conclusion, we applied CT-based measures to identify structural variables of the proximal femur and the acetabulum that are associated with low-energy acetabular fractures. The technique used enabled a true morphometric analysis in 3D. Our analyses had excellent repeatability for all variables analyzed. We found that elderly subjects with NSA values that are smaller than normal are prone to low-energy acetabular fractures. Although we found no statistically significant association of acetabular morphology with fracture cases, we did observe some over-coverage. Further work is needed to fully understand the etiology of low-energy acetabular fractures in the elderly. 


\section{Acknowledgments}

This study was financially supported by CINOP Global through the NICHE project NICHE/ETH/246 funded by EP-Nuffic (Netherlands). Dr. Esa Liukkonen is acknowledged for his contribution to the gathering of images from PACS of Oulu University Hospital. 


\section{References}

[1] R. Firoozabadi, W.W. Cross, J.C. Krieg, M.L.J. Chip Routt, Acetabular Fractures in the Senior Population- Epidemiology, Mortality and Treatments, Arch Bone Jt Surg. 5 (2017) 96-102. doi:10.22038/abjs.2016.7933.

[2] D. Butterwick, S. Papp, W. Gofton, A. Liew, P.E. Beaulé, Acetabular Fractures in the Elderly, J Bone Joint Surg Am. 97 (2015) 758-68. doi:10.1007/978-1-4614-3511-2_11.

[3] A. Laird, J.F. Keating, Acetabular fractures: A 16-Year Prospective Epidemiological Study, J Bone Joint Surg (Br). 87-B (2005) 969-973. doi:10.1302/0301-620X.87B7.16017.

[4] P. Kannus, J. Parkkari, S. Niemi, H. Sievänen, Low-Trauma Pelvic Fractures in Elderly Finns in 1970-2013, Calcif Tissue Int. 97 (2015) 577-580. doi:10.1007/s00223-015-0056-8.

[5] T.A. Ferguson, R. Patel, M. Bhandari, J.M. Matta, Fractures of the acetabulum in patients aged 60 years and older: An Epidemiological And Radiological Study, J Bone Joint Surg (Br). 92-B (2010) 250-257. doi:10.1302/0301-620X.92B2.22488.

[6] P.E. Beaule, D.B. Griffin, J.M. Matta, The Levine anterior approach for total hip replacement as the treatment for an acute acetabular fracture, J Orthop Trauma. 18 (2004) 623-629.

[7] G. Anastopoulos, D. Chissas, J. Dourountakis, P.G. Ntagiopoulos, E. Magnisalis, A. Asimakopoulos, T.A. Xenakis, Computer-assisted three-dimensional correlation between the femoral neck-shaft angle and the optimal entry point for antegrade nailing, Injury. 41 (2010) 300-305. doi:10.1016/j.injury.2009.09.007.

[8] J. Thevenot, J. Hirvasniemi, M. Finnilä, P. Pulkkinen, V. Kuhn, T. Link, F. Eckstein, T. Jämsä, S. Saarakkala, Trabecular homogeneity index derived from plain radiograph to evaluate bone quality, J Bone Miner Res. 28 (2013) 2584-2591. doi:10.1002/jbmr.1987.

[9] P. Pulkkinen, F. Eckstein, E.M. Lochmüller, V. Kuhn, T. Jämsä, Association of geometric factors and failure load level with the distribution of cervical vs. trochanteric hip fractures, $\mathbf{J}$ Bone Miner Res. 21 (2006) 895-901. doi:10.1359/jbmr.060305.

[10] C.K. Boese, J. Jostmeier, J. Oppermann, J. Dargel, D.H. Chang, P. Eysel, P. Lechler, The neck shaft angle: CT reference values of 800 adult hips, Skeletal Radiol. 45 (2016) 455-463. doi:10.1007/s00256-015-2314-2.

[11] J.A. Hanson, A.L. Kapron, K.M. Swenson, T.G. Maak, C.L. Peters, S.K. Aoki, Discrepancies in measuring acetabular coverage: revisiting the anterior and lateral center edge angles., $\mathrm{J}$ Hip Preserv Surg. 2 (2015) 280-6. doi:10.1093/jhps/hnv041.

[12] S. Ogata, H. Moriya, K. Tsuchiya, T. Akita, M. Kamegya, M. Someya, Acetabular Cover in Congenital Dislocation of The Hip, J Bone Joint Surg (Br). 72-B (1990) 190-196.

[13] A. Mittal, J.D. Bomar, M.E. Jeffords, M.T. Huang, D.R. Wenger, V. V. Upasani, Defining the lateral edge of the femoroacetabular articulation: correlation analysis between 
radiographs and computed tomography, $\mathbf{J}$ Child Orthop. 10 (2016) 365-370. doi:10.1007/s11832-016-0768-y.

[14] S. Anda, S. Svenningsen, L.G. Dale, P. Benum, The acetabular sector angle of the adult hip determined by computed tomography, Acta Radiol. 27 (1986) 443-447. doi:10.1177/028418518602700415.

[15] T. Schwarz, A. Benditz, H.R. Springorum, J. Matussek, G. Heers, M. Weber, T. Renkawitz, J. Grifka, B. Craiovan, Assessment of pelvic tilt in anteroposterior radiographs by means of tilt ratios, Archives of Orthopaedic and Trauma Surgery. 138 (2018) 1045-1052. doi:10.1007/s00402-018-2931-z.

[16] T. Schwarz, M. Weber, M. Wörner, T. Renkawitz, J. Grifka, B. Craiovan, Central X-ray beam correction of radiographic acetabular cup measurement after THA: an experimental study, Int J CARS. 12 (2017) 829-837. doi:10.1007/s11548-016-1489-x.

[17] S.W. Higgins, E.M. Spratley, R.A. Boe, C.W. Hayes, W.A. Jiranek, J.S. Wayne, A Novel Approach for Determining Three-Dimensional Acetabular Orientation: Results from Two Hundred Subjects, J Bone Joint Surg Am. 96 (2014) 1776-1784. doi:10.2106/JBJS.L.01141.

[18] D. Suzuki, S. Nagoya, H. Takashima, K. Tateda, T. Yamashita, Three-dimensional orientation of the acetabulum, Clin Anat. 30 (2017) 753-760. doi:10.1002/ca.22945.

[19] H. Zhang, Y. Wang, S. Ai, X. Chen, L. Wang, K. Dai, Three-dimensional acetabular orientation measurement in a reliable coordinate system among one hundred Chinese, PLoS One. 12 (2017) 1-12. doi:10.1371/journal.pone.0172297.

[20] J. Partanen, T. Jämsä, P. Jalovaara, Influence of the upper femur and pelvic geometry on the risk and type of hip fractures, J Bone Miner Res. 16 (2001) 1540-1546. doi:10.1359/jbmr.2001.16.8.1540.

[21] H. van Bosse, J.H. Wedge, P. Babyn, How Are Dysplastic Hips Different? A Threedimensional CT Study, Clin Orthop Relat Res. 473 (2015) 1712-1723. doi:10.1007/s11999014-4103-y.

[22] M. Tannast, M.S. Hanke, G. Zheng, S.D. Steppacher, K.A. Siebenrock, What Are the Radiographic Reference Values for Acetabular Under- and Overcoverage?, Clin Orthop Relat Res. 473 (2015) 1234-1246. doi:10.1007/s11999-014-4038-3.

[23] K. Tallroth, J. Lepistö, Computed tomography measurement of acetabular dimensions: normal values for correction of dysplasia., Acta Orthop. 77 (2006) 598-602. doi:10.1080/17453670610012665.

[24] R. Ganz, M. Leunig, K. Leunig-Ganz, W.H. Harris, The etiology of osteoarthritis of the hip: An integrated mechanical concept, Clin Orthop Relat Res. 466 (2008) 264-272. doi:10.1007/s11999-007-0060-z.

[25] P. Pulkkinen, J. Partanen, P. Jalovaara, T. Jämsä, BMD T-score discriminates trochanteric 
fractures from unfractured controls, whereas geometry discriminates cervical fracture cases from unfractured controls of similar BMD, Osteoporos Int. 21 (2010) 1269-1276. doi:10.1007/s00198-009-1070-3.

[26] P. Pulkkinen, J. Partanen, P. Jalovaara, M.T. Nieminen, T. Jämsä, Combination of radiograph-based trabecular and geometrical parameters can discriminate cervical hip fractures from controls in individuals with BMD in non-osteoporotic range, Bone. 49 (2011) 290-294. doi:10.1016/j.bone.2011.04.016.

[27] D.W. Murray, The definition and measurement of acetabular orientation., J Bone Joint Surg (Br). 75 (1993) 228-232. doi:Article.

[28] I. Sharp, Acetabular dysplasia the acetabular angle, J Bone Joint Surg (Br). 43-B (1961) 268272. doi:10.1017/CBO9781107415324.004.

[29] P. Pulkkinen, C.C. Glüer, T. Jämsä, Investigation of differences between hip fracture types: A worthy strategy for improved risk assessment and fracture prevention, Bone. 49 (2011) 600-604. doi:10.1016/j.bone.2011.07.022.

[30] C. Ripamonti, L. Lisi, M. Avella, Femoral neck shaft angle width is associated with hipfracture risk in males but not independently of femoral neck bone density, Br J Radiol. 87 (2014) 1-5. doi:10.1259/bjr.20130358.

[31] R.Y. Wang, W.H. Xu, X.C. Kong, L. Yang, S.H. Yang, Measurement of acetabular inclination and anteversion via CT generated 3D pelvic model, BMC Musculoskelet Disord. 18 (2017) 1-7. doi:10.1186/s12891-017-1714-y.

[32] A. Milcan, A. Yildiz, V. Öztuna, M.M. Eskandari, G. Şahin, F. Kuyurtar, The anterior center edge angle: A study of 102 volunteers, Joint Bone Spine. 71 (2004) 221-223. doi:10.1016/S1297-319X(03)00121-0.

[33] D. Miyasaka, Y. Sakai, S. Ibuchi, H. Suzuki, N. Imai, N. Endo, Sex- and age-specific differences in femoral head coverage and acetabular morphology among healthy subjectsderivation of normal ranges and thresholds for abnormality, Skeletal Radiol. 46 (2017) $523-$ 531. doi:10.1007/s00256-017-2583-z.

[34] H. Ito, T. Matsuno, T. Hirayama, H. Tanino, Y. Yamanaka, A. Minami, Three-dimensional computed tomography analysis of non-osteoarthritic adult acetabular dysplasia, Skeletal Radiol. 38 (2009) 131-139. doi:10.1007/s00256-008-0601-x.

[35] V. Chadayammuri, T. Garabekyan, M.-K. Jesse, C. Pascual-Garrido, C. Strickland, K. Milligan, O. Mei-Dan, Measurement of lateral acetabular coverage: a comparison between CT and plain radiography, J Hip Preserv Surg. 2 (2015) 63. doi:10.1093/jhps/hnv063.

[36] R. Letournel, E., Judet, Fractures of the acetabulum, 2nd ed., Library of Congress, 1993. 


\section{Figure Legends}

Figure 1. Flowchart of the study showing final study datasets after elimination processes for acetabular and proximal femur geometry measurements. CT images of femurs and acetabula of insufficient quality were eliminated from both geometry studies. Two-sided acetabular fractures were eliminated from acetabular geometry analysis only. 
Figure 2. Geometrical measurements of the proximal femur shown in an isometric view. (O) is the center of the sphere fitted around the femoral head, (C-D) is femoral head diameter (FHD), (A-B) is femoral neck axis length-a (FNALa), (O-B) is femoral neck axis length-b (FNALb), and $(l)$ is femoral shaft axis. Neck shaft angle (NSA) is the angle between $(l)$ and (A-B). (E-F) is neck shaft width (NSW). 
Figure 3. Re-orientation of the 3D model into a new coordinate system. (a) Isometric view of the 3D model. Points A and B are right and left bilateral anterior superior iliac spines (ASIS), and points $\mathrm{C}$ and D are right and left pubic tubercles (PT). (b) Top view of the 3D model before (light green) and after (turquoise) alignment. APP-1 (solid line) is the original position of the anterior pelvic plane (APP) (dotted line) before alignment. TPP indicates the trans-pelvic plane and MSP the mid-sagittal plane. AI is acetabular inclination and Ace AV is acetabular anteversion. 
Figure 4. Acetabular geometry measurements. (a) Acetabular angle of Sharp (AA) and lateral center edge angle (LCEA), (b) Anterior and posterior sector angles (ASA and PSA), (c) Anterior and posterior center edge angles (ACEA and PCEA). $(\mathrm{O})$ is the center of the femoral head, $(\mathrm{A})$ is lateral edge of the acetabular roof, (B) is bony ridges of the acetabular fossa, (C) and (D) are anterior and posterior margins on TPP respectively, and (E) and (F) are anterior and posterior margins on MSP respectively. AA was measured between a horizontal line through (B) and a line connecting (A) and (B). LCEA was measured between a line connecting (A) and (O) and a vertical line. ASA, PSA, ACEA and PCEA were measured between a vertical line through $(\mathrm{O})$ and a line from $(\mathrm{O})$ to acetabular margins $(\mathrm{C}),(\mathrm{D}),(\mathrm{E})$ and $(\mathrm{F})$ respectively. 


\section{Tables}

Table 1. Intrarater repeatability and interrater reliability of proximal femur and acetabular geometry measurements. Intraclass correlations coefficients (ICC) at 95\% CI for randomly selected cases $(N=20,10$ fractures and 10 controls).

\begin{tabular}{lcccc}
\hline \multirow{2}{*}{ Variables } & \multicolumn{2}{l}{ Intrarater } & \multicolumn{2}{c}{ Interrater } \\
\cline { 2 - 5 } & ICC & $95 \%$ CI & ICC & $95 \%$ CI \\
\hline NSA & 0.840 & $0.668-0.931$ & 0.885 & $0.775-0.949$ \\
FNALb & 0.919 & $0.831-0.965$ & 0.900 & $0.804-0.956$ \\
FNALa & 0.935 & $0.863-0.972$ & 0.913 & $0.830-0.962$ \\
NSW & 0.916 & $0.824-0.964$ & 0.947 & $0.892-0.977$ \\
FHD & 0.958 & $0.882-0.984$ & 0.974 & $0.929-0.990$ \\
AceAV & 0.986 & $0.969-0.994$ & 0.991 & $0.982-0.996$ \\
AI & 0.959 & $0.913-0.983$ & 0.978 & $0.955-0.990$ \\
AA & 0.967 & $0.921-0.986$ & 0.979 & $0.956-0.991$ \\
LCEA & 0.978 & $0.938-0.991$ & 0.985 & $0.965-0.994$ \\
ACEA & 0.982 & $0.951-0.993$ & 0.988 & $0.969-0.995$ \\
PCEA & 0.981 & $0.957-0.992$ & 0.989 & $0.971-0.994$ \\
PSA & 0.970 & $0.916-0.989$ & 0.981 & $0.954-0.992$ \\
ASA & 0.979 & $0.941-0.992$ & 0.986 & $0.964-0.994$ \\
\hline
\end{tabular}


Table 2. A summary of proximal femur and acetabular geometry variables. Mean (SD) and P-values in the fracture and control groups of entire data and gender specific values are shown.

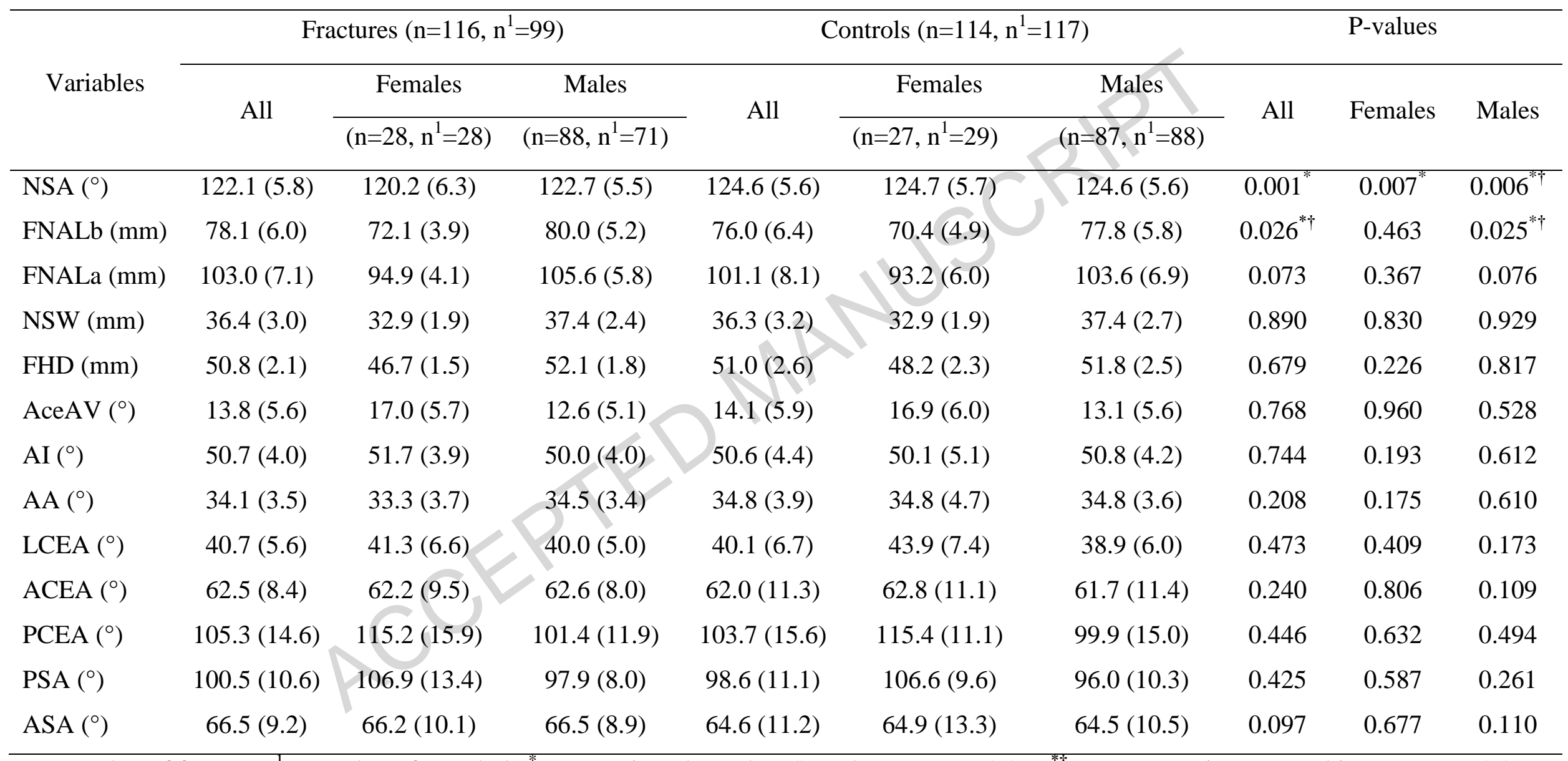

$\mathrm{n}=$ number of femurs, $\mathrm{n}^{1}=$ number of acetabula, ${ }^{*}$ Parametric Independent Samples t-test $(p<0.05),{ }^{* \dagger}$ Nonparametric Mann-Whitney test $(p<0.05)$. 
Table 3. Bivariate Pearson's linear correlation coefficients between proximal femur and acetabular geometry variables

\begin{tabular}{|c|c|c|c|c|c|c|c|c|c|c|c|c|}
\hline Variables & AceAV & AI & AA & LCEA & ACEA & PCEA & PSA & ASA & FHD & NSW & FNALa & FNALb \\
\hline NSA & 0.100 & 0.066 & 0.045 & $-0.209^{* * *}$ & -0.086 & -0.116 & -0.115 & $-0.252^{* 2 x}$ & 0.032 & -0.006 & 0.070 & 0.032 \\
\hline FNALb & $-0.290^{* *}$ & -0.061 & -0.045 & -0.019 & 0.089 & $-0.216^{* *}$ & $-0.215^{* *}$ & $0.185^{* *}$ & $0.466^{* *}$ & $0.525^{* *}$ & $0.962^{* *}$ & \\
\hline FNALa & $-0.300^{* *}$ & -0.041 & -0.008 & -0.008 & 0.061 & $-0.263^{* *}$ & $-0.260^{* * *}$ & $0.162^{*}$ & $0.657^{* *}$ & $0.660^{* *}$ & & \\
\hline NSW & $-0.229^{* *}$ & 0.094 & $0.195^{* *}$ & $-0.301^{* *}$ & -0.059 & $-0.299^{* *}$ & $-0.296^{* *}$ & -0.001 & $0.778^{* *}$ & & & \\
\hline FHD & $-0.188^{* *}$ & 0.039 & 0.099 & $-0.283^{* *}$ & -0.127 & $-0.357^{* *}$ & $-0.343^{* *}$ & -0.049 & & & & \\
\hline ASA & $-0.578^{* *}$ & $-0.258^{* *}$ & $-0.164^{* *}$ & $0.513^{* *}$ & $0.708^{* *}$ & -0.062 & $-0.195^{* *}$ & & & & & \\
\hline PSA & $0.629^{* *}$ & $0.244^{* *}$ & 0.098 & $0.299^{* * *}$ & $0.160^{* *}$ & $0.833^{* *}$ & & & & & & \\
\hline PCEA & $0.634^{* *}$ & $0.341^{* *}$ & $0.173^{* *}$ & $0.274^{* *}$ & $-0.237^{* *}$ & & & & & & & \\
\hline ACEA & $-0.619^{* *}$ & $-0.391^{* *}$ & $-0.245^{* *}$ & $0.492^{* *}$ & & & & & & & & \\
\hline
\end{tabular}

${ }^{* *}$ Correlation is significant at $p<0.01$ level (2-tailed). 
Table 4. Bivariate Pearson's linear correlation coefficients $R$ between right and left sides of proximal femur and acetabular geometry variables and their mean value differences (P-values). Analysis was done on a randomly selected subset of 19 (5 female, 14 male) control subjects.

\begin{tabular}{lccccccccccccc}
\hline Variables & NSA & FNALb & FNALa & NSW & FHD & AceAV & AI & AA & LCEA & ACEA & PCEA & PSA & ASA \\
\hline$R$ & $0.532^{* *}$ & $0.965^{* *}$ & $0.919^{* *}$ & $0.905^{* *}$ & $0.946^{* *}$ & $0.884^{* *}$ & $0.842^{* *}$ & $0.814^{* * *}$ & $0.797^{* *}$ & $0.845^{* *}$ & $0.851^{* *}$ & $0.825^{* *}$ & $0.888^{* *}$ \\
P-value $^{\S}$ & 0.05 & 0.774 & 0.860 & 0.978 & 0.773 & 0.437 & 0.711 & 0.751 & 0.733 & 0.396 & 0.757 & 0.851 & 0.432
\end{tabular}

${ }^{* *}$ Correlation is significant at $p<0.01$ level (2-tailed). ${ }^{\S}$ Mean value differences calculated for each variable after checking normality distribution. 


\section{Authors' roles:}

Study concept and design: RG, SS, JL, TJ. Data collection: RG, IL. Data analysis and validation: RG, JH. Data interpretation: RG, JH, TJ. Writing - original draft: RG. Writing - review \& editing: All authors. Approving final version of manuscript: All authors. 


\section{Highlights}

- Our aim was to identify proximal femur and acetabular geometry structural risk factors of lowenergy acetabular fractures in elderly subjects.

- Geometry measurements using clinical two-dimensional radiography can be difficult and prone to errors.

- Three-dimensional computed tomography provides a more reliable measurement tool.

- We compared fracture cases to their age and gender matched controls.

- Proximal femur geometry was found to be associated with low-energy acetabular fractures. 


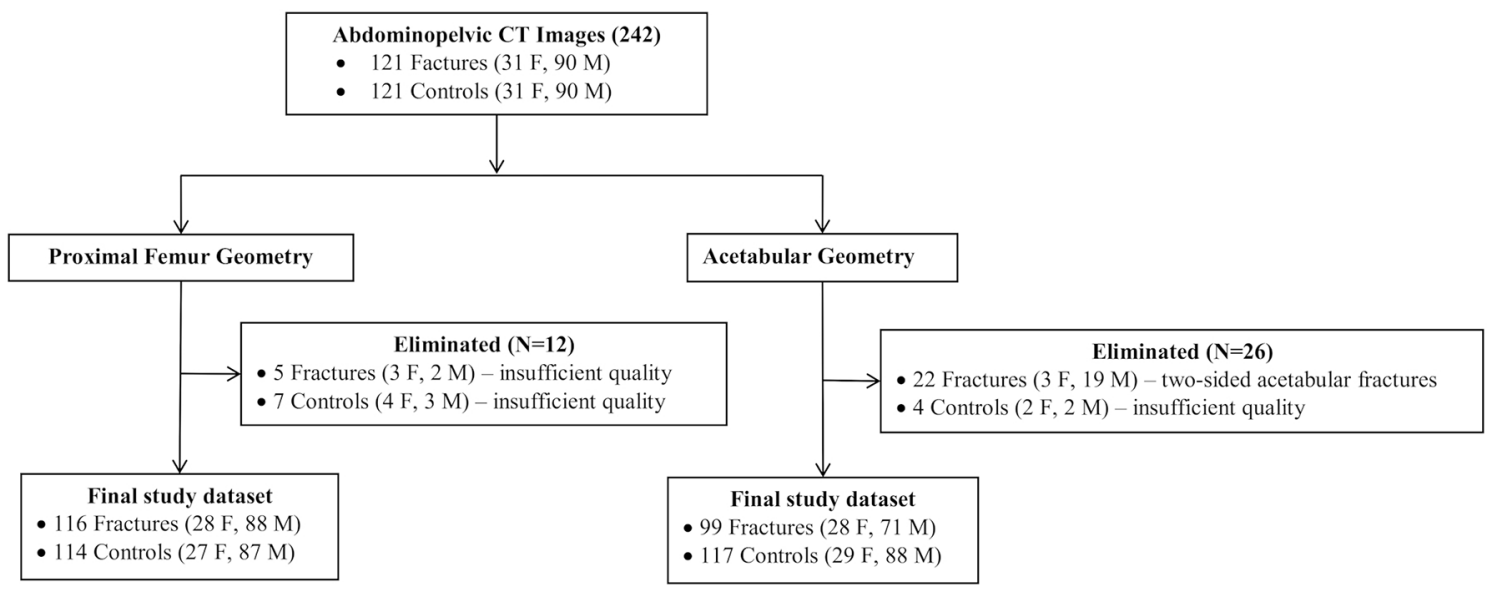

Figure 1 


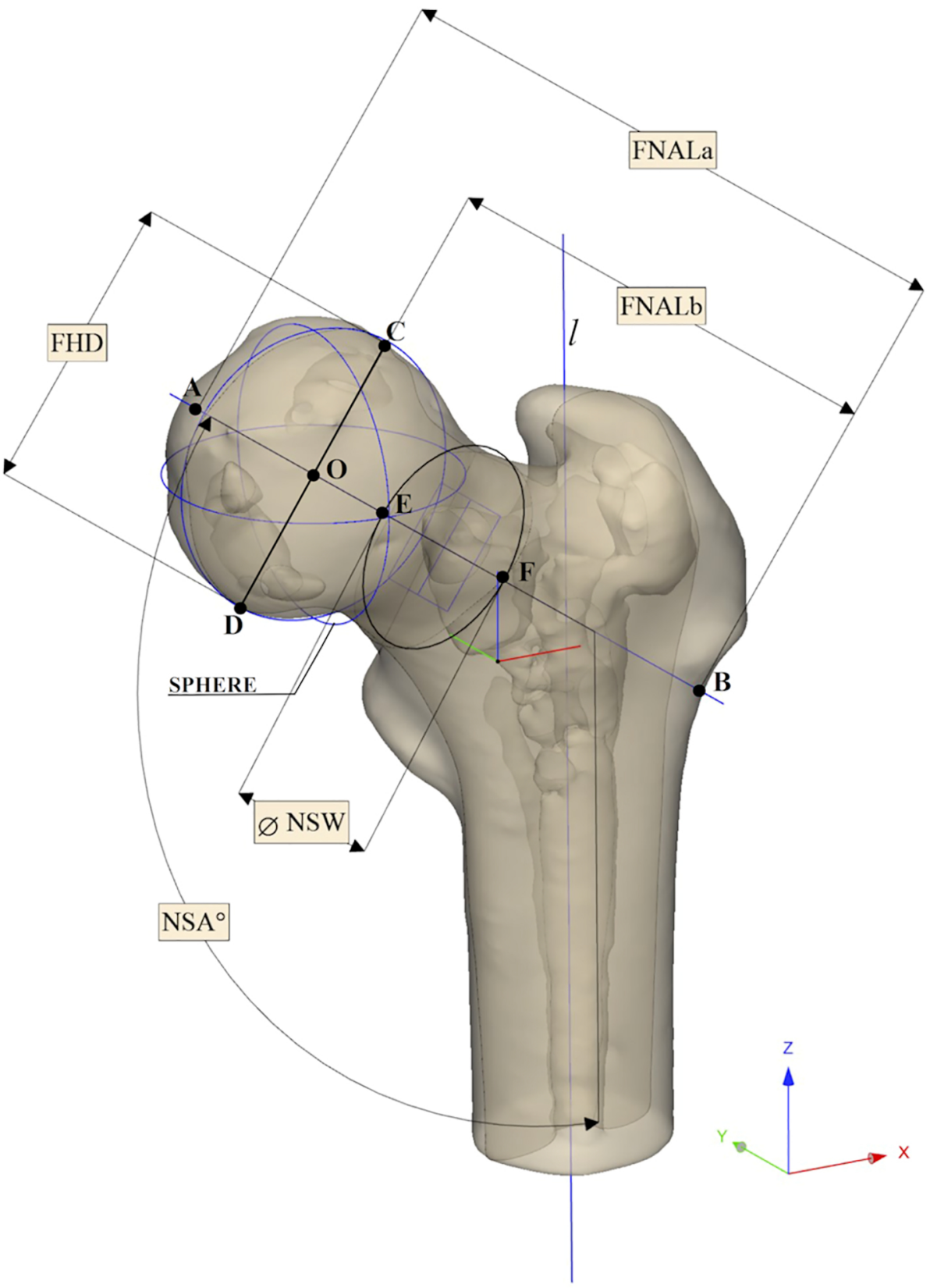

Figure 2 


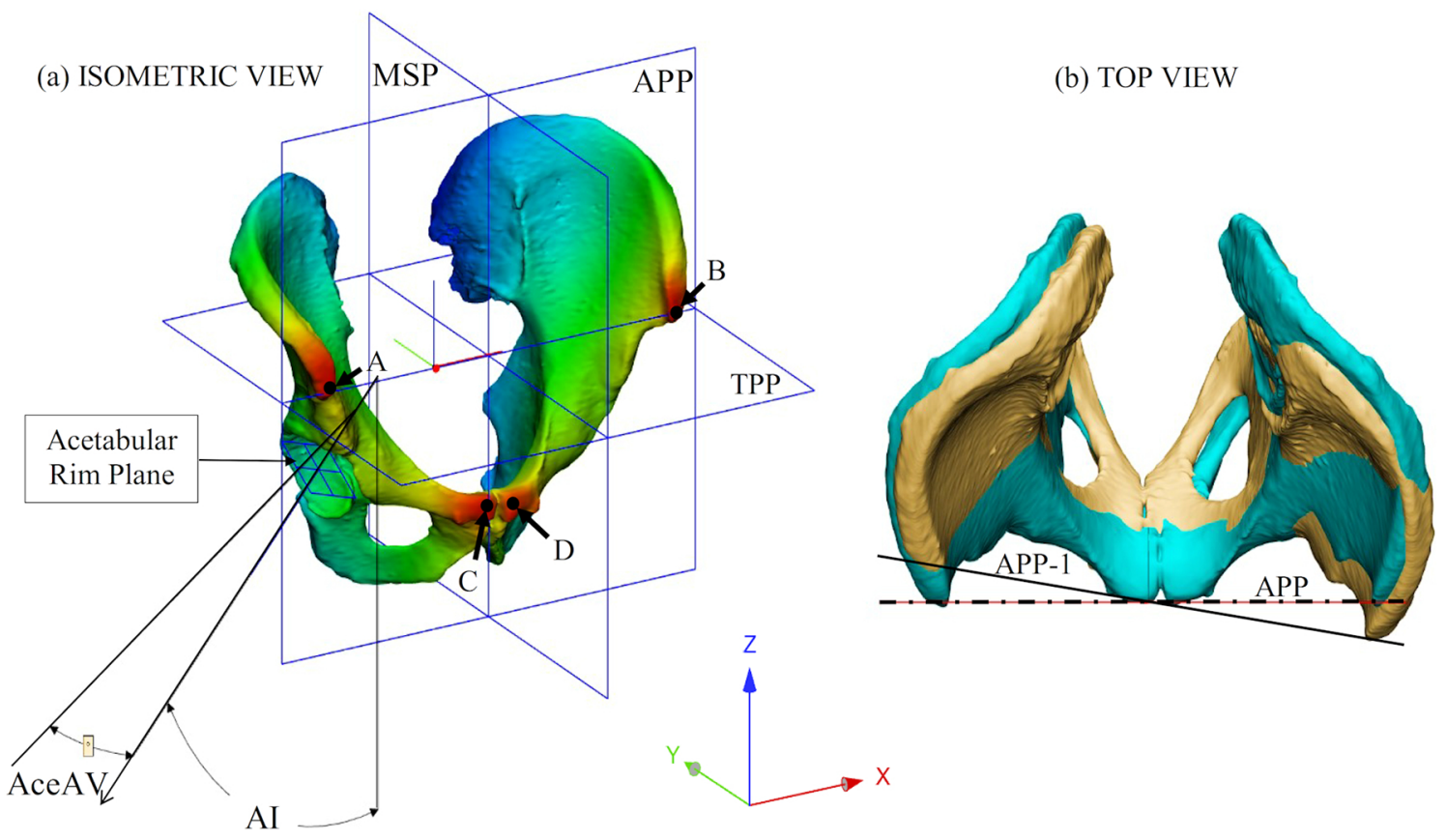

Figure 3 

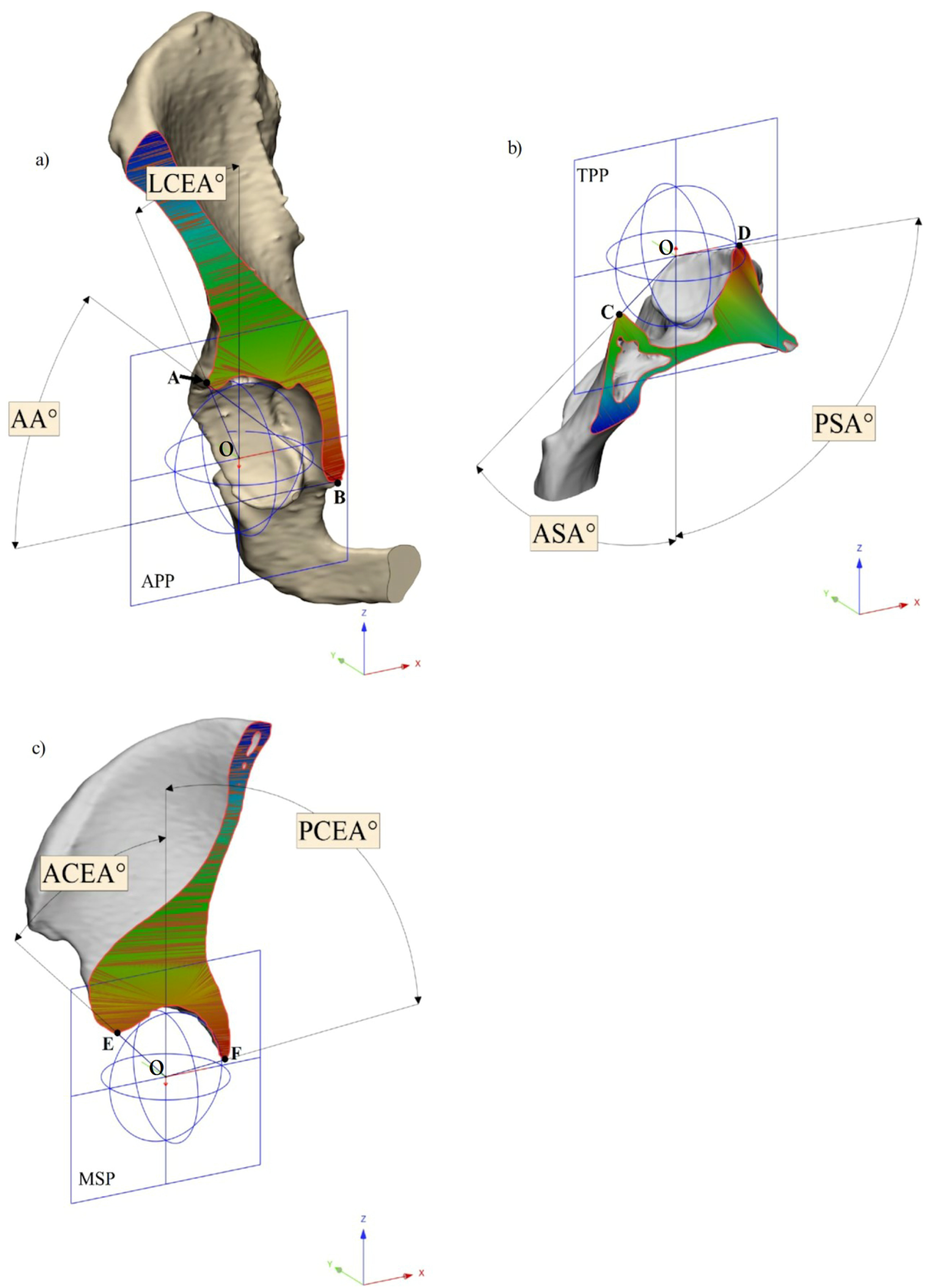